\title{
Impacto clínico del estroncio y del zinc en los procesos de regeneración ósea y del cartílago, aproximación desde la biología hacia la clínica. Revisión de literatura
}

\section{Clinical impact of strontium and zinc on bone and cartilage regeneration processes, an approach from biology to the clinic. Literature review}

\begin{abstract}
Resumen
Muchos trastornos sistémicos como la artritis o la osteoporosis son patologías responsables de las alteraciones crónicas de la articulación temporomandibular, creando así un problema a largo plazo que afectan la calidad de vida de aquellas personas que las padecen. Actualmente no existe tratamiento curativo para dichas patologías, aunque sí de tipo paliativo, que en muchas ocasiones tienen efectos secundarios en el tiempo o una limitación en su efectividad y eficacia, por lo que se hace necesario buscar alternativas con la implementación de terapias regenerativas para el tratamiento de aquellas enfermedades que afectan el sistema musculoesquelético. En muchos estudios se discute sobre el papel fundamental que cumple el zinc y el estroncio en la génesis de tejido tanto cartilaginoso como óseo, así como la relevancia de incorporarlos con diversos biomateriales en procedimientos de regeneración, sin embargo, este tema no es claro aún y requieren más atención por parte del clínico. El objetivo de este artículo es determinar la función cumplen el zinc y el estroncio en los procesos de regeneración del hueso y el cartílago desde una visión molecular y celular aplicada a la práctica clínica, con el fin de obtener nuevas alternativas en el tratamiento de diversas patologías y alteraciones musculoesqueléticas que mejoren las condiciones de salud oral de los pacientes, además de, contar con la revisión que nos aproxime a comprender los mecanismos de acción de diferentes moléculas que incorporadas a los biomateriales compatibles con el tejido duro y blando mejoren las condiciones biológicas para la regeneración.
\end{abstract}

Palabras clave: Regeneración; Hueso; Cartílago; Zinc; Estroncio; Biomateriales (fuente: DeCS BIREME).

\begin{abstract}
Many systemic disorders such as arthritis or osteoporosis are pathologies responsible of temporomandibular joint chronic dysfunctions, thus creating a long-term problem that affects life's quality of those who suffer from them. Currently there is no curative treatment for these pathologies, although there is a palliative treatment, which in many cases have side effects over time or a limitation in their effectiveness and efficacy, so it is necessary to look for alternatives with the implementation of regenerative therapies for treatment of those diseases that affect musculoskeletal system. In many studies the fundamental role of zinc and strontium in genesis of both cartilaginous and bone tissue is discussed, as well as the relevance of incorporating them with various biomaterials in
\end{abstract}

\section{Artículo de Revisión}

Samuel Urbano del Valle 1,a, María Catalina Castaño Granada ${ }^{1,6}$

${ }^{1}$ Universidad de Antioquia, Medellín, Colombia. a Especialista en Estomatología, Cirugía Oral y Maxilofacial.

${ }^{\mathrm{b}}$ Especialista en Periodoncia.

\section{Correspondencia:}

Samuel Urbano del Valle: samuel.urbano@udea.edu.co Facultad de Odontología, Universidad de Medellín, Campus de la Salud. Medellín - Antioquia.

ORCID: 0000-0002-5773-8642

\section{Coautor:}

María Catalina Castaño Granada: catalina.castano@ udea.edu.co

ORCID: 0000-0001-5497-6437

\section{Editora:}

María Eugenia Guerrero Acevedo

Universidad Nacional Mayor de San Marcos, Perú.

Conflicto de intereses: los autores declaran no tener conflictos de interés.

\section{Financiamiento: ninguno.}

\section{Recibido: 03/09/20}

Aceptado: 02/02/21

Publicado: 01/04/21 
regeneration procedures, however, this issue is not clear yet and requires more attention from the clinician. The objective of this article is to determine function of zinc and strontium in regeneration processes of bone and cartilage from a molecular and cellular perspective applied to clinical practice, in order to obtain new alternatives in the treatment of various pathologies and musculoskeletal alterations that improve the oral health conditions of patients. In addition, this review brings us closer to understanding the mechanisms of action of different molecules that when incorporated into biomaterials compatible with hard and soft tissue improve the biological conditions for the regeneration.

Keywords: Regeneration; Bone; Cartilage; Zinc; Strontium; Biomaterials (source: MeSH NLM).

\section{Introducción}

El desequilibrio entre la reabsorción y la formación que se presenta en enfermedades tales como la osteogénesis imperfecta, la osteoartritis, la artritis reumatoide, la enfermedad de Paget, entre muchas otras, afecta de manera compleja y muchas veces severa a las personas que la padecen, situación que crea la necesidad de evaluar diversas estrategias biológicas necesarias para la reparación y regeneración de los tejidos afectados, generando un desafío para el clínico al implementar terapias que permitir restablecer el equilibrio o sustituir el tejidos óseos y cartílago afectado en diferentes patologías generalmente de tipo crónico ${ }^{1-4}$.

La osteoporosis es una de las enfermedades de tipo musculoesquelético más común, que consiste principalmente en el deterioro de la masa ósea a causa de la reabsorción y por consiguiente en el deterioro que se produce paulatinamente con el aumento de la edad, todo esto sumado a los factores epigenéticos tales como la predisposición genética, el estrés, la obesidad, el sexo o el estilo de vida. En la actualidad, el tratamiento de dicha enfermedad se centra en suplir el calcio y la vitamina D, así como el uso de ciertos medicamentos, como los bifosfonatos, terapias de reemplazo hormonal, el ranelato de estroncio o los inhibidores de RANK ${ }^{5-7}$.

Los estudios más recientes revelan que, aunque la formación de hueso está determinada por la vía de señalización Wnt/b-catenina canónica, la reabsorción osteoclástica está mediada por la vía RANKL/osteoprogeterina (OPG)/RANK y su función en enfermedades que afecta el metabolismo óseo se encuentra alteradas, al existir un desbalance entre la activación de osteoblastos formadores del hueso y la función osteoclástica en la reabsorción ${ }^{8-10}$.

El propósito fundamental de las terapias de regeneración ha sido siempre el de restablecer la salud al paciente, por lo que el clínico dirige su atención en implementar tratamientos tanto para el reemplazo de órganos, células y tejidos creando andamios biológicamente activos con materiales biocompatibles y bioactivos, por lo tanto el objetivo de este artículo es presentar cuál es el papel biológico que tienen el Zinc y el Estroncio en las estrategias de regeneración ósea y cartilaginosa ${ }^{11-13}$.

\section{Métodos}

Se realizó una búsqueda electrónica médico-dental en las bases de datos Pubmed, ScienceDirect, Scopus, Medline, EBSCO y Google Schoolar desde 1985 hasta junio del 2020. Se utilizó la siguiente estrategia de búsqueda: ((Zinc and Strontium) OR (regenerative materials) OR (biomaterials) OR (regenerative medicine) OR (scaffolds) OR (multifunctional materials) OR (tissue engineering) OR (engineering for bone) OR (bioactive materials) OR (bone graft materials) OR (synthetic materials)) AND ((guided bone regeneration) OR (guided bone augmentation) OR (bone augmentation) OR (osseous repair) OR (graft integration)). La búsqueda electrónica se complementó con una búsqueda manual tomando en consideración la lista de referencias de las publicaciones incluidas.

Selección de estudios: criterios de elegibilidad. Se verificó la relevancia de los títulos y resúmenes de los artículos obtenidos para luego descargar el texto completo de los artículos pertinentes cuando era posible; de lo contrario, solo se obtuvieron los resúmenes electrónicos.

Se consideraron los siguientes criterios de inclusión:

- Existencia de un grupo prueba y de un grupo control.

- Ensayos clínicos aleatorizados en humanos.

- Ensayos aleatorizados en animales.

- Análisis mediante microscopía electrónica de barrido.

- Evaluación de composición elemental por EDX.

- Evaluaciones con histomorfometría.

- Análisis mediante microtomografía computarizada.

Se consideraron los siguientes criterios de exclusión:

- Estudios con descripciones ambiguas del procedimiento.

- Estudios con resultados de un solo grupo evaluado.

- Estudios en elementos finitos, pilotos, reportes de caso y artículos de discusión.

\section{Estroncio y zinc}

Se entiende que para la síntesis de ciertas moléculas estructurales de las células y de la matriz ósea existe un cofactor indispensable como el zinc ( $\mathrm{Zn})$, que en el 
tejido óseo participa en la formación al inducir la función osteoblástica e inhibir la reabsorción medida por los osteoclastos para la homeostasis del sistema musculoesquelético. El zinc además, cumple una función indispensable en la estructura de la matriz ósea y participa activamente en el metabolismo de la matriz de cartílago con la acción de las metaloproteinasas (MMP) 36-33 dependientes de Zn y la expresión de SOX9 y el gen del cartílago II (Figura). Así mismo, cuando se producen desequilibrios asociados a la disminución del Zn se presentan retrasos en el crecimiento gonadal ${ }^{7-9}$.

En la literatura se reporta ampliamente la búsqueda de opciones en los tratamiento de regeneración de los tejido perdidos o afectados basadas en la aplicación de biomateriales y algunas terapias más recientes incluyen el uso del estroncio ( $\mathrm{Sr}$ ), el cual está conformado por ácido ranélico orgánico mezclado con dos átomos de $\mathrm{Sr}$, cuyos efectos se encuentran en la actualidad bien estudiados en aquellos pacientes con osteoporosis u otras enfermedades del sistema musculoesquelético ya que estimula la formación no sólo de tejido óseo sino también de cartílago (Figura) ${ }^{10}$.

Varios autores explican que muchos pacientes con fracturas óseas y alteraciones articulares a nivel de la rodilla mejoran indudablemente después de la administración de biomateriales con $\mathrm{Sr}$ ya que dicho compuesto se asocia con el crecimiento en proporciones adecuadas de osteocitos para la conformación de tejido óseo y cartílago, demostrando así la formación de hueso trabecular a nivel de la tibia en osteoartritis, y en osteonecrosis de los maxilares ${ }^{11}$.
Se conoce, además, que la absorción de estroncio $(\mathrm{Sr})$ es inducida por la vitamina $\mathrm{D}$, y a su vez es inhibida por la acción del calcio, participando activamente en las funciones metabólicas de la matriz ósea y el cartílago e infiriendo su importancia en la terapia regenerativa. En la actualidad se reconoce que el ranelato de Sr puede estar incluido como componente de diferentes biomateriales para inducir la formación de hueso nuevo y promover la reparación de fracturas en mujeres posmenopáusicas, el cual estimula la formación de hueso en dosis bajas ${ }^{12}$.

Una de las áreas de mayor impacto en el uso de biomateriales de regeneración ha sido la implantología oral. Los implantes dentales y aquellos de uso ortopédico están constituidos en mayor medida por una serie de aleaciones y como componente principal por el titanio, con diferentes grados de pureza que le proporcionan excelentes propiedades de biocompatibilidad y estabilidad mecánica y química ${ }^{13,14}$.

Estudios recientes demuestran que los cambios o alteraciones en la superficie de los implantes mejoran las condiciones biológicas, moleculares y celulares que permiten que, los tejidos duros y blandos se adapten a la nueva estructura sobre el hueso, es por ello que recientemente se comenzó a incluir el elemento Sr como SrTi03/TiO276 dentro de los componentes de muchos de estos dispositivos odontológicos. Otro punto relevante en estas instancias, es la reacción del sistema inmune a los biomateriales que son usados en conjunto con el Sr ${ }^{9,15,16}$.

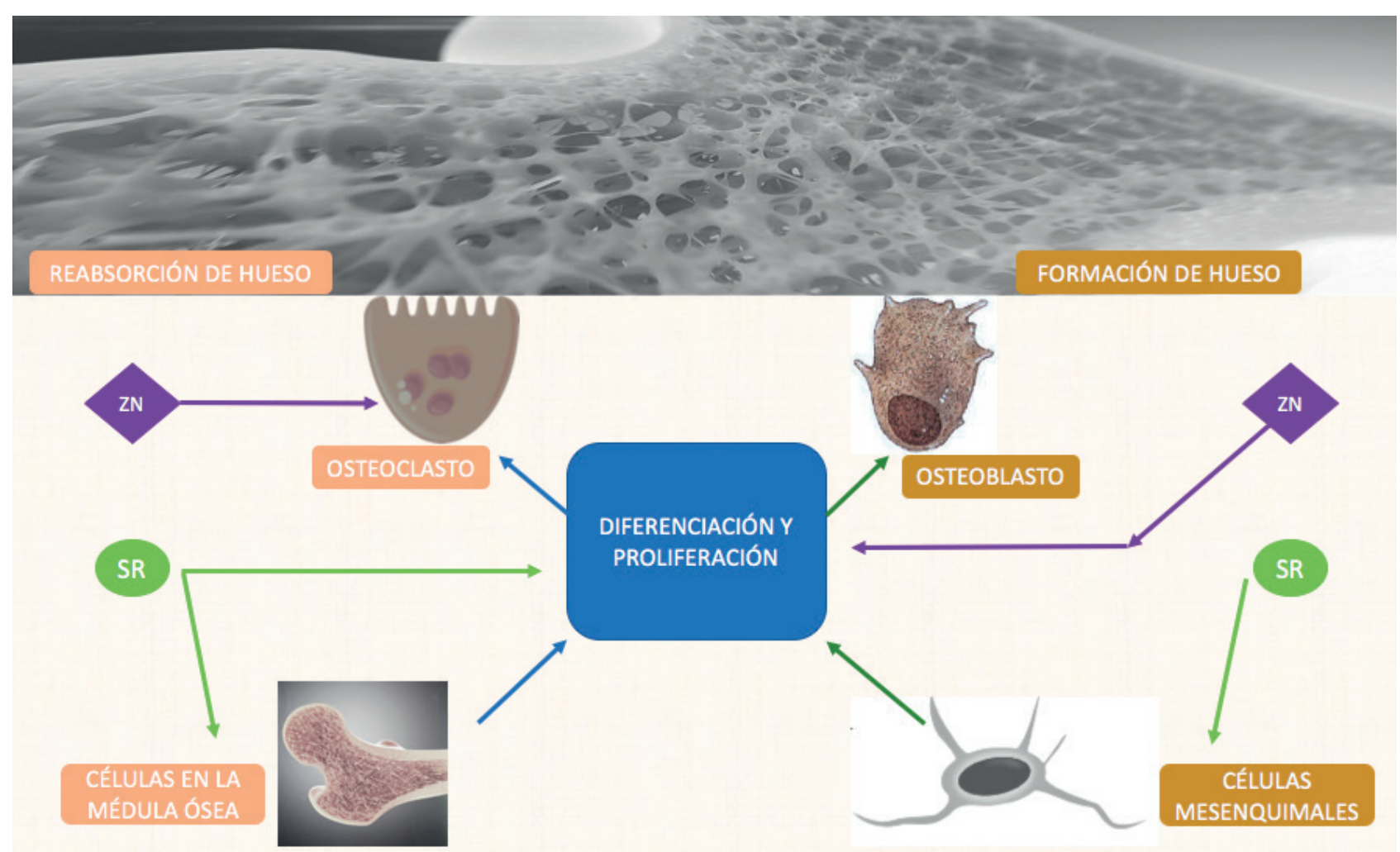

Figura. Mecanismo de acción del estroncio y del zinc en el hueso 
Teniendo en cuenta las propiedades osteoinductoras del titanio y las propiedades osteogénicas del calcio $(\mathrm{Ca})$ y estroncio (Sr), Ekuzo y colaboradores, realizaron un estudio donde tomaron cinco de Ti / DOPA / SA / Sr 1 ( $1 \%$ en peso de $\mathrm{Sr}$ ), Ti, Ti / DOPA y Ti / DOPA / SA / Sr5 (5\% en peso de Sr) y las analizaron con MG-63 células, y con Ti / DOPA / SA, con el fin de valorar la eficiencia para la cicatrización ósea y la formación de hueso con un biomaterial prometedor como el alginato de sodio, y la utilidad de éste asociado al Ca y al Sr para la regeneración de tejido óseo. Los resultados obtenidos demuestran la posibilidad de utilizar Sr y dopamina en la superficie del titanio para modificar la superficie del implante ${ }^{16-18}$.

Imitar las propiedades biológicas y físicoquímicas del tejido óseo y el cartílago, que induzca la capacidad de diferenciarse, proliferar e integrarse es un proceso complejo y temporo-espacialmente controlado. Sin embargo, en la actualidad se reportan diversos materiales osteoconductores que permiten constituirse en andamios biológicamente estables y pueden estar formados de diferentes sustitutos óseos tales como, materiales sintéticos, polímeros naturales o la combinación de éstos ${ }^{19}$.

En la ingeniería de tejidos, el uso de materiales sintéticos como el vidrio bioactivo, constituyen una excelente alternativa, ampliamente utilizada y reportada en la literatura que permite a su vez incorporar otros materiales como el estroncio ( $\mathrm{Sr}$ ) dentro de sus componentes, conservando sus propiedades y siendo un material biocompatible, y con actividad biológica capaz de recrear las condiciones precisas para la regeneración ósea ${ }^{19-21}$.

En varios estudios se aplicaron combinaciones diferentes, en las que sustituyeron el óxido de calcio $(\mathrm{CaO})$ por $\mathrm{Sr}$, haciendo uso de células del estroma de la médula ósea, usando técnicas como la de enfriamiento por fusión para poder mezclar dos o más componentes ${ }^{22,23}$.

A nivel del sistema estomatognático se llevaron a cabo estudios en los que se simula la presencia de células madre de órganos dentarios deciduos, con el fin de recrear las condiciones biológicas y físicas para el desarrollo y formación de los dientes en los maxilares, teniendo en cuenta que la matriz ósea está formada por hidroxiapatita y por otros componentes orgánicos, cómo el colágeno, los cuales supeditan la conducta de las células. Por todo ello, en dicho estudio, se sumergen nanofibras en fosfato de $\mathrm{Sr}$ y matriz ósea, obteniendo de esta forma tres compuestos, nanofibras de Poli e-caprolactona (PCL) + 0,8\% Sr, nanofibras de PCL recubiertas con Sr y nanofibras de PCL, compuestos con los que imitaron las células madre de los dientes deciduos. Finalmente se logra crear un biomaterial usando la hidroxiapatita que se encuentra en la matriz ósea para poder implantarlo y no generar reacciones adversas ${ }^{24,25}$.

Por otro lado, se plantean propuestas innovadoras realizadas en animales en Nueva Zelanda, en donde se fusionan dos sistemas de cementos óseos y vidrios con actividad biológica inductora conformados por líquido y polvo (monómero MMA + polimetacrilato de metilo
+ peróxido de benzoilo y circonia- $\mathrm{ZrO} 2-)$, además de fosfato tricálcico, $8,5 \%$ tanto de hidroxiapatita como de carbonato de estroncio y una solución de fosfato de hidrógeno al 4\% con el propósito de inducir la formación ósea obteniendo una alta tasa de éxito del 83,5\% 26,27.

En otro estudio realizado en Nueva Zelanda se mezclaron un compuesto híbrido con $\mathrm{Zn}$ y Sr para formar un hidrogel de alginato reticulado reforzado con selenio (Se) e hidroxiapatita en forma de microesferas dopadas con $\mathrm{Sr}$, que fueron aplicadas directamente en un defecto producido por la resección quirúrgica de un condrosarcoma, y se pudo observar con éxito la conformación de un entramado andamiaje de tejido óseo ${ }^{27}$.

Finalmente, se sabe que es de crucial importancia la aplicación de aquellos biomateriales que están conformados por la unión del Zn y del Sr para el desarrollo y crecimiento de células progenitoras, pues está bien demostrado que el uso de $\mathrm{Zn}$ evita la pérdida ósea ya que limita el proceso de resorción y mejora la formación de los tejidos óseos y cartilaginosos, conduciendo de esta forma a un impacto netamente terapéutico en las patologías osteoarticulares.

\section{Discusión}

Las terapias de regeneración que se basan en el uso de estroncio y zinc, han llamado en gran medida la atención de diferentes investigadores, gracias a sus características, a su fácil acceso, a la seguridad y a la alta estabilidad que proporcionan, potenciando a los diferentes biomateriales en los que se usan en conjunto, características de inducción, conducción y génesis tanto de tejidos óseos como de tejidos cartilaginosos ${ }^{13-15,28}$.

Múltiples estudios in vitro e in vivo han valorado gran variedad de nuevas terapias, así como la implementación de estrategias de regeneración con Sr y Z. Estudios in vitro realizados en promedio con $55 \%$ de células humanas y $45 \%$ células de origen animal demuestran la capacidad de formación ósea determinada por la proliferación y morfología de las células encontradas en los resultados cuantitativos de PCR, así como la expresión genética de gran variedad de moléculas tales como, el factor de transcripción Runx2, la fosfatasa alcalina, la osteocalcina, la osteopontina, el colágeno tipo I y la proteína morfogenética ósea 2 relacionadas con los mecanismos formación y desarrollo del tejido óseo ${ }^{15,29}$.

Los resultados de los diferentes estudios evaluados tanto en humanos como en animales demuestran que la regeneración del tejido óseo fue de un $77,9 \%$, en una relación de 9 sobre 10 y con formación de hueso nuevo en un $89,1 \%$ en el restante de casos, porcentajes que reflejan que la regeneración ósea es casi completa, además de presentar características muy notables tanto en osteoconducción como en biocompatibilidad, obteniendo resultados muy prometedores que fueron evaluados en el fémur y en la tibia ${ }^{16-18,30}$.

De los estudios anteriormente mencionados, todos combinaron $\mathrm{Sr}$ y $\mathrm{Zn}$ excepto en uno in vivo, en el que utilizaron Sr para promover la regeneración ósea, y finalmente 
concluyen que existe una amplia evidencia que tanto el $\mathrm{Sr}$ como el Zn no sólo pueden generar inhibición de la formación de osteoclastos o inducir la formación de hueso, sino que además inducen la expresión de genes relacionados con la diferenciación y maduración de las células precursoras de hueso y con síntesis de moléculas que promueven e inician la mineralización de la matriz ósea ${ }^{28-29}$.

Es importante destacar que, aunque los diferentes materiales evaluados en los estudios analizados en la presente revisión demuestran que la incorporación Sr y Zn mejoran la formación ósea e inhiben la reabsorción, el mecanismo de acción aún no es del todo claro. Sin embargo, los investigadores relacionan los hallazgos en la regeneración y neoformación ósea y del tejido cartilaginoso con los mecanismos biológicos necesarios para este proceso se presente, como los son, la angiogénesis, el depósito de minerales en la matriz extracelular, la adhesión celular, la inmunomodulación, la respuesta regulada de los macrófagos y fundamentalmente la protección de los osteoblastos en su ciclo vital ${ }^{30-31}$.

Otros estudios encuentran diversas dificultades asociadas al vehículo de cationes bivalentes en algunos biomateriales, no obstante, existen algunas investigaciones que han mostrado ser muy prometedoras en cuanto a mejoras al vehículo o transporte, en las que sustituyen el ácido ranélico por derivados de Vitamina B de Sr y $\mathrm{Zn}{ }^{16,29-31}$.

\section{Conclusión}

Actualmente, existen muchos artículos científicos que demuestran la función del estroncio y del zinc en las estrategias de regeneración tisular, ya sea propiciando la formación de nuevo tejido a partir de la diferenciación de células madre, de cartílago y hueso, como también en la inhibición en la tasa de reabsorción del tejido neoformado.

No obstante, aún existen algunas limitaciones en los estudios y los resultados son controversiales, por lo que se debe trabajar e investigar más en el área de la odontología y en diferentes líneas de investigación que promuevan el desarrollo de nuevos biomateriales, donde se incorporen diversas moléculas que mejoren las características y la biocompatibilidad con los tejidos receptores, teniendo en cuenta los hallazgos previos del comportamiento y los excelentes resultados obtenidos con el uso de algunos minerales como el estroncio y el zinc en las estrategias de regeneración ósea y de cartílago.

\section{Agradecimiento}

A la Universidad de Antioquia por permitirnos avanzar en conocimientos a través de la investigación.

\section{Referencias bibliográficas}

1. Florencio-Silva R, Rodrigues G, Sasso-Cerri E, Simoes MJ, Cerri PS. Biology of Bone Tissue: Structure, Function, and Factors That Influence Bone Cells. BioMed Res Int. 2015;2015:421746. DOI: 10.1155/2015/421746.
2. Rodan GA, Martin TJ. Therapeutic approaches to bone diseases. Science. 2000;289(5484):1508-14. DOI: 10.1126/science.289.5484.1508.

3. Neves N, Linhares D, Costa G, Ribeiro CC, Barbosa MA. In vivo and clinical application of strontium-enriched biomaterials for bone regeneration: A systematic review. Bone Joint Res. 2017;6(6):366-75. DOI: 10.1302/2046-3758.66.BJR-2016-0311.R1.

4. Gogakos Al, Cheung MS, Bassett JD, Williams GR. Bone signaling pathways and treatment of osteoporosis. Expert Rev Endocrinol Metab. 2009;4(6):639-50. DOI: 10.1586/eem.09.38.

5. Colombo F, Sampogna G, Cocozza G, Guraya SY, Forgione A. Regenerative medicine: Clinical applications and future perspectives. J Microsc Ultrastruct. 2017;5(1):1-8. DOI: 10.1016/j.jmau.2016.05.002.

6. Lee HY, Lie D, Lim KS, Thirumoorthy T, Pang SM. Strontium ranelate-induced toxic epidermal necrolysis in a patient with post-menopausal osteoporosis. Osteoporos Int. 2009;20(1):161-2. DOI: 10.1007/s00198-0080677-0.

7. Fukada T, Yamakasi S, Nishida K, Mukarami M, Hirano T. Zinc homeostasis and signaling in health and diseases: Zinc signaling. J Biol Inorg Chem. 2011;16(7):1123-34. DOI: $10.1007 / \mathrm{s} 00775-011-0797-4$.

8. Demoor M, Ollitrault D, Gomez-Leduc T, Bouyoucef M, Hervieu M, Fabre H, et al. Cartilage tissue engineering: molecular control of chondrocyte differentiation for proper cartilage matrix reconstruction. Biochim Biophys Acta. 2014;1840(8):2414-40. DOI: 10.1016/j. bbagen.2014.02.030.

9. Rahaman MN, Day DE, Bal BS, Fu Q, Jung SB, Bonewald LF. Bioactive glass in tissue engineering. Acta Biomater. 2011;7(6):2355-73. DOI: 10.1016/j.actbio.2011.03.016.

10. Al Qaysi M, Walters NJ, Foroutan F, Owens GJ, Kim HW, Shah R, et al. Strontium- and calcium-containing, titanium-stabilised phosphate-based glasses with prolonged degradation for orthopaedic tissue engineering. J Biomater Appl. 2015;30(3):300-10. DOI: $10.1177 / 0885328215588898$.

11. Kargozar S, Lotfibakhshaiesh N, Ai J, Mozafari M, Milan PB, Hamzehlou $S$ et al. Strontium- and cobalt-substituted bioactive glasses seeded with human umbilical cord perivascular cells to promote bone regeneration via enhanced osteogenic and angiogenic activities. Acta Biomater. 2017;58:502-14. DOI: 10.1016/j.actbio.2017.06.021.

12. Birgani ZT, Malhotra A, van Blitterswijk CA, Habibovic P. Human mesenchymal stromal cells response to biomimetic octacalcium phosphate containing strontium. J Biomed Mater Res A. 2016;104(8):1946-60. DOI: 10.1002/jbm.a.35725

13. Shi C, Hu B, Guo L, Cao P, Tian Y, Ma J et al. Strontium Ranelate Reduces the Fracture Incidence in a Growing Mouse Model of Osteogenesis Imperfecta. J Bone Miner Res. 2016;31(5):1003-14. DOI: 10.1002/jbmr.2770.

14. Yu W, Sun TW, Qi C, Ding Z, Zhao H, Zhao S et al. Evaluation of zinc-doped mesoporous hydroxyapatite microspheres for the construction of a novel biomimetic scaffold 
optimized for bone augmentation. Int J Nanomedicine. 2017;12:2293-306. DOI: 10.2147/IJN.S126505.

15. Zhu D, Su Y, Young ML, Ma J, Zheng Y, Tang L. Biological Responses and Mechanisms of Human Bone Marrow Mesenchymal Stem Cells to $\mathrm{Zn}$ and Mg Biomaterials. ACS Appl Mater Interfaces. 2017;9(33):27453-61. DOI: 10.1021 /acsami.7b06654.

16. Taylor BA, Bezuhly M, Brace M, Carter M, Hong P. Effect of strontium citrate on bone consolidation during mandibular distraction osteogenesis. Laryngoscope. 2017;127(7):E212-18. DOI: 10.1002/lary.26623.

17. Fernández JM, Molinuevo MS, Sedlinsky C, Schurman L, Cortizo AM, McCarthy AD. Strontium ranelate prevents the deleterious action of advanced glycation end products on osteoblastic cells via calcium channel activation. Eur J Pharmacol. 2013;706(1-3):41-7. DOI: 10.1016/j.ejphar.2013.02.042.

18. Carmo ABX, Sartoretto SC, Novellino ATN, Granjeiro JM, Miguel FB, Maia JC et al. Alveolar bone repair with strontium- containing nanostructured carbonated hydroxyapatite. J Appl Oral Sci. 2018;26:e20170084. DOI:10.1590/1678-7757-2017-0084.

19. Li JJ, Ebied M, Xu J, Zreiqat H. Current Approaches to Bone Tissue Engineering: The Interface between Biology and Engineering. Adv Healthc Mater. 2018;7(6):e1701061. DOI:10.1002/adhm.201701061.

20. Neves N, Linhares D, Costa G, Ribeiro CC, Barbosa MA. In vivo and clinical application of strontium-enriched biomaterials for bone regeneration: A systematic review. Bone Joint Res. 2017;6(6):366-75. DOI: 10.1302/2046-3758.66.BJR-2016-0311.R1.

21. Manolagas SC. Wnt signaling and osteoporosis. Maturitas. 2014;78(3):233-7. DOI: 10.1016/j.maturitas.2014.04.013.

22. Querido W, Rossi A, Farina M. The effects of strontium on bone mineral: A review on current knowledge and microanalytical approaches. Micron. 2016;80:122-34. DOI: $10.1016 /$ j.micron.2015.10.006.

23. Boyd D, Carroll G, Towler MR, Freeman C, Farthing $\mathrm{P}$, Brook IM. Preliminary investigation of novel bone graft substitutes based on strontium-calcium-zinc-silicate glasses. J Mater Sci Mater Med. 2009;20(1):413-20. DOI:10.1007/s10856-008-3569-0.
24. Santocildes ME, Crawford A, Hatton PV, Goodchild RL, Reany IM, Miller CA. The osteogenic response of mesenchymal stromal cells to strontium-substituted bioactive glasses. J Tissue Eng Regen Med. 2015;9(5):619-31. DOI: $10.1002 /$ term.2003.

25. Kumar PK, Mahato A, Kundu B, Nandi SK, Mukherjee P, Datta $S$ et al. Influence of single and binary doping of strontium and lithium on in vivo biological properties of bioactive glass scaffolds. Sci Rep. 2016;6:32964. DOI: 10.1038/srep32964.

26. Al Qaisy M, Walters NJ, Foroutan F, Owens GJ, Kim HW, Shah R et al. Strontium- and calcium-containing, titanium-stabilised phosphate-based glasses with prolonged degradation for orthopaedic tissue engineering. J Biomater Appl. 2015;30(3):300-10. DOI: $10.1177 / 0885328215588898$.

27. Robinson L, Salma-Ancane K, Stipniece L, Meenan BJ, Boyd AR. The deposition of strontium and zinc Co-substituted hydroxyapatite coatings. J Mater Sci Mater Med. 2017;28(3):51. DOI:10.1007/s10856-017-5846-2.

28. Ehret C, Launais RA, Sagardoy T, Siadous R, Bareille R, Rey $S$ et al. Strontium-doped hydroxyapatite polysaccharide materials effect on ectopic bone formation. PLoS One. 2017;12(9):e0184663. DOI: 10.1371/journal. pone. 0184663 .

29. Shepherd JH, Shepherd DV, Best SM. Substituted hydroxyapatites for bone repair. J Mater Sci Mater Med. 2012;23(10):2335-47. doi:10.1007/s10856-012-4598-2.

30. Cheng D, Liang Q, Li Y, Fan J, Wang G, Pan H et al. Strontium incorporation improves the bone-forming ability of scaffolds derived from porcine bone. Colloids Surf B Biointerfaces. 2018;162:279-87. DOI: 10.1016/j. colsurfb.2017.11.070.

31. Jiao J, Kaplan LD, Zreigat H. Scaffold-based regeneration of skeletal tissues to meet clinical challenges. Journal of materials chemistry B. 2014;2(42):7272-306. DOI: 10.1039/c4tb01073f. 